\title{
Geschlechtertheorie und Gleichstellungspraxis - eine theoretische und eine empirische Perspektive
}

\section{Zusammenfassung}

Seit einigen Jahren finden in der hochschulischen Gleichstellungsarbeit Entwicklungen statt, die als Professionalisierungsprozesse gedeutet werden. Während das traditionelle Professionsverständnis für die Analyse der Gleichstellungsarbeit wenig zielführend ist, bieten dynamische Konzepte der Professionalisierung eine fruchtbare Forschungsperspektive. Zentral für die Professionalisierung ist dabei das an der jeweiligen Wissenschaft eines Tätigkeitsbereichs orientierte ExpertInnenwissen. Mit der zunehmenden Implementierung der Frauen- und Geschlechterforschung steht der hochschulischen Gleichstellungsarbeit wissenschaftliches Geschlechterwissen zur Verfügung. Der Transfer dieses Wissens auf die Gleichstellungspraxis stößt allerdings auf Hürden, für deren Überwindung man bessere Kenntnis des Arbeitsalltags und des Bedarfs nach theoretischem Geschlechterwissen von GleichstellungspraktikerInnen braucht. Erste Aufschlüsse hierzu liefern zwei quantitative und qualitative Erhebungen mit Amts- und StelleninhaberInnen der hochschulischen Gleichstellungsarbeit. Diese weisen auf das Vorhandensein eines beachtlichen geschlechtertheoretischen Unterbaus bei den GleichstellungsakteurInnen hin sowie auf eine Bereitschaft, diesen zu nutzen. Die Nutzbarmachung erfolgt bisher allerdings unsystematisch. Hier könnten Anknüpfungspunkte für eine weitere Professionalisierung der Gleichstellungsarbeit liegen.

\section{Schlüsselwörter}

Gleichstellung, Hochschule, Professionalisierung, Frauen- und Geschlechterforschung, Genderkompetenz

\section{Summary \\ Theory and practice of gender equality work - a theoretical and an empirical perspective}

For several years a professionalization process has been taking place in gender equality politics. This paper discusses the use of sociological concepts in regard to the professionalization of gender equality work in higher education. While the traditional understanding of professions is unrewarding when it comes to an analysis of gender equality work, we refer to dynamic concepts of professionalization. These concepts focus on expert knowledge as a major criterion of professionalization processes. The implementation of gender studies in German universities has the potential to provide scientific expert knowledge for the work of gender quality practitioners. But the transfer of this theoretical knowledge to practical work comes up against many obstacles. Better knowledge about the practitioners' work routines and their demand for theoretical gender knowledge is needed to overcome those obstacles. Two surveys presented in this article - one quantitative, the other qualitative - present first insights into how that theory-practice transfer can be improved. The surveys addressed gender equality practitioners at universities. Results indicate that there are many practitioners who were educated in gender studies and who are willing to use this knowledge for their work although there is as yet no systematic way of doing that. Changing this fact might support the further professionalization of gender equality politics.

Keywords

gender equality, university, professionalization, gender studies, gender competence 


\section{Einleitung}

Hochschulleitungen sind angesichts erhöhter externer Anforderungen an die Gleichstellungspolitik in Form der Forschungsorientierten Gleichstellungsstandards der DFG, des Professorinnenprogramms und der Berücksichtigung von Gleichstellungsaspekten in der Exzellenzinitiative sowie der neuen Steuerungsmechanismen unter Druck geraten, mehr personelle und finanzielle Ressourcen für die Erhöhung der Chancengleichheit von Männern und Frauen an Hochschulen ${ }^{1}$ zu investieren (Blome et al. 2013; Macha/Gruber/Struthmann 2011; Zimmermann 2003). Die Implementierung neuer Steuerungsmechanismen, auch bekannt unter dem Begriff „New Public Management“, hat in den letzten Jahren das Augenmerk auf Professionalisierungsprozesse im Hochschulmanagement gelenkt (Krücken/Blümel/Kloke 2010; Schneijderberg/Merkator 2011). Obwohl Gleichstellungsarbeit als Teil des administrativen Hochschulmanagements gesehen werden kann, stand dieser Bereich bisher selten im Fokus der Untersuchungen. Dabei hat Meuser (2005a) bereits vor einigen Jahren Professionalisierungsprozesse bei GenderexpertInnen identifiziert, die er vor allem auf die Implementierung der Ansätze Gender Mainstreaming und Managing Diversity in der Gleichstellungspolitik zurückführt. Zu beobachten sei in diesem Zuge eine erhöhte Nachfrage nach einem neuen Wissenstyp bzw. einem neuen Kompetenzprofil, nämlich dem Genderwissen bzw. der Genderkompetenz. Ob die Professionalisierung der Gleichstellungspolitik, die laut Meuser an einer Akademisierung der Wissensvermittlung und Vorläufern einer Standesorganisation erkennbar ist, in eine Profession mündet, lässt er offen (Meuser 2005a).

Im Rahmen des Forschungsprojektes „Hochschulische Gleichstellungsstrukturen im Wandel" $(\mathrm{HGiW})^{2}$ wird die Professionalisierung von GleichstellungsakteurInnen an Hochschulen unter einer professionssoziologischen Perspektive untersucht. Der vorliegende Beitrag, der sich auf dieses Projekt bezieht, befasst sich mit der Rolle theoretischwissenschaftlichen Geschlechterwissens für die Professionalisierung der Gleichstellungsarbeit an Hochschulen. Nach einer kurzen Erläuterung des Professionsbegriffs und -verständnisses wird die hochschulische Gleichstellungsarbeit aus professionssoziologischer Perspektive beleuchtet. Daran anknüpfend werden für dieses Feld anwendbare Modelle der Professionalisierung zusammengefasst, die vor allem das ExpertInnenwissen als bedeutendes Kriterium für Professionalisierung fokussieren. Im anschließenden empirischen Teil werden Ergebnisse einer quantitativen Online-Befragung sowie einer qualitativen Interviewstudie mit Amts- und StelleninhaberInnen der hochschulischen Gleichstellungsarbeit vorgestellt. Die beiden Erhebungen geben Aufschluss über die tatsächliche Bedeutung, die theoretischem Geschlechterwissen durch GleichstellungspraktikerInnen zukommt, über die Selbstwahrnehmung der Befragten bezüglich ihrer Genderexpertise sowie über das Verständnis des Begriffs Genderexpertise.

1 Der Begriff Hochschule umfasst im vorliegenden Beitrag Universitäten, staatliche und private Fachhochschulen bzw. Hochschulen für angewandte Wissenschaften sowie künstlerische und pädagogische Hochschulen.

2 Das Projekt wurde von 2011 bis 2013 aus Mitteln des BMBF und des Europäischen Sozialfonds der Europäischen Union unter dem Förderkennzeichen 01FP1080/81 gefördert und am Kompetenzzentrum Frauen in Wissenschaft und Forschung CEWS in der GESIS durchgeführt. 
Diesem Beitrag liegt bei der Betrachtung der Gleichstellungsarbeit ein analytisches Verständnis des Professionalisierungsbegriffs (siehe dazu Kleimann 2011) ${ }^{3}$ zugrunde und keinesfalls eine normative Unterscheidung zwischen professioneller oder weniger professioneller Arbeit von GleichstellungsakteurInnen.

\section{Gleichstellungsarbeit aus professionssoziologischer Sicht}

Um die Entwicklungsprozesse, die in den letzten Jahren in der hochschulischen Gleichstellungsarbeit $\mathrm{zu}$ beobachten waren, professionssoziologisch einordnen zu können, werden im Folgenden zunächst das klassische Professionsverständnis und anschließend dynamische Modelle der Professionalisierung auf ihre Anwendung für die Gleichstellungsarbeit hin geprüft. Im Fokus steht das ExpertInnenwissen, speziell das wissenschaftliche Geschlechterwissen, als entscheidendes Kriterium für Professionalisierungsprozesse.

\subsection{Das klassische Professionsverständnis}

Die klassische professionssoziologische Literatur richtet den Professionsbegriff vor allem an den Urprofessionen wie Jura und Medizin aus. Das Professionsverständnis ist dabei zwar uneinheitlich, allerdings meist kriteriengeleitet. Zusammengefasst muss eine Profession folgende Kriterien erfüllen: Es existiert ein gesellschaftlich relevanter Problembereich mit einem dazugehörigen Handlungs- und Erklärungswissen. Des Weiteren wird auf einen gesellschaftlichen Zentralwert Bezug genommen, mit dem sich ein hohes Einkommen und Privilegien öffentlich rechtfertigen lassen. Eine akademische Spezialausbildung ist für Professionen eine wichtige Voraussetzung, da diese abstraktes, für die professionelle Berufstätigkeit unverzichtbares Wissen zur Verfügung stellt. Damit ist es einer Profession möglich, einen spezifischen Wissens- und Problembereich exklusiv zu besetzen (Mieg 2003, 2006). Kleimann spricht in diesem Zusammenhang von einer „weltbildprägenden professionsspezifischen Sozialisation“ (Kleimann 2011: 215). Zudem existiert ein Berufsverband bzw. eine berufsständische Vertretung, welche neben der Organisation der Profession auch deren Selbstkontrolle durch die Auferlegung von Ethikcodes und Verhaltensregeln dient.

Zwei der drei zentralen Professionsmerkmale sind damit bereits benannt: (ExpertInnen-)Wissen und Autonomie. Das ExpertInnenwissen ist dabei als Voraussetzung für die Autonomie zu sehen. Erst durch das Vorhandensein einer spezifischen Expertise kann sich eine Berufsgruppe gegenüber anderen Berufsgruppen abgrenzen und schließlich ein Zuständigkeitsmonopol in Bezug auf ein thematisches Feld für sich beanspruchen. Dieser Anspruch einer professionellen Kompetenz und Zuständigkeit ist ohne eine

3 Kleimann (2011) unterscheidet bei der Untersuchung von Professionalisierungsprozessen zwischen der analytischen und der normativen Ebene. Der analytische Professionsbegriff orientiert sich an einem Merkmalset, anhand dessen der Professionsgrad einer Berufsgruppe ermittelt wird. Der Begriff ist deskriptiv und somit nicht wertend. Durch Verwendung des analytischen Begriffs ist es möglich, die Professionalisierung einer Berufsgruppe zu untersuchen, ohne eine Wertung bezüglich ihrer "guten" oder "effizienten" Arbeit vorzunehmen. 
Untermauerung durch wissenschaftliche Erkenntnisse und facheigene Methoden nicht erfolgreich durchsetzbar, da es Kompetenzen bedarf, die LaiInnen nicht ohne Weiteres zur Verfügung stehen. Neben dem akademischen Wissen setzt sich das ExpertInnenwissen auch aus Berufswissen im Sinne von Erfahrungswissen und spezifischem Problemlösungs- und Deutungswissen zusammen (Meyer 2000). Auf der individuellen Ebene grenzt die Autonomie ExpertInnen von LaiInnen ab, auf der kollektiven Ebene die spezialisierte Berufsgruppe von der Außenwelt. Eine Profession gilt weiterhin dann als autonom, wenn sie sich durch eine Unabhängigkeit im beruflichen Handeln von organisierten gesellschaftlichen Kräften auszeichnet, sodass eine eigenständige Entscheidungsfindung in Bezug auf die Arbeitspraktiken möglich ist. Außerdem ist sie zur Selbstrekrutierung in der Lage, d. h. zur Personalselektion durch Professionsmitglieder (Evetts 2003; Meyer 2000). Neben Wissen und Autonomie ist die KlientInnenorientierung das dritte Merkmal, welches eine Profession konstituiert. So beschreibt Schütze (1992) die Profession als einen ,relativ abgegrenzten Orientierungs- und Handlungsbereich, in welchem sowohl wissenschaftlich als auch praktisch ausgebildete Berufsexperten gesellschaftlich lizenzierte Dienstleistungen für ihnen per gesellschaftlichem Mandat anbefohlene Klienten bzw. Abnehmer vollbringen“ (Schütze 1992: 135).

\subsection{Gleichstellungsarbeit - eine Semi-Profession?}

Bei der Anwendung professionssoziologischer Konzepte auf die Arbeit von Frauen- und Gleichstellungsbeauftragten kommt Nigges-Gellrich zu dem Schluss, dass dieses Vorhaben nur möglich erscheint, ,wenn und sofern der Betrachter von dem tradierten Professionsverständnis abrückt und den Weg zur Profession nicht an der Überwindung von Merkmalshürden misst“" (Nigges-Gellrich 2008: 33). Orientiere man sich an den Kriterien traditioneller Professionskonzepte, müsse man die Gleichstellungsarbeit als SemiProfession einstufen (Meyer 2000; Nigges-Gellrich 2008). Für die Gleichstellungsarbeit gibt es keine einheitliche standardisierte Ausbildung, die durch staatliche Instanzen zertifiziert wird. Folglich verfügen GleichstellungsakteurInnen über keine gemeinsame Wissensbasis oder ein einheitliches Kompetenzprofil. Eine klare Abgrenzung zwischen GleichstellungsexpertInnen und Gleichstellungs-,,LaiInnen“ gibt es nicht. Außerdem ist die Gleichstellungsarbeit in der Regel Teil der Verwaltung einer Organisation, womit das Kriterium der Autonomie nicht erfüllt werden kann.

\section{3 "Neue" Modelle der Professionalisierung}

Die Anwendung des kriteriengeleiteten Professionsbegriffs auf die heutige Berufswelt stößt auf mehrere Probleme. Denn das klassische Professionsverständnis schließt nicht nur GleichstellungspraktikerInnen, sondern prinzipiell die meisten modernen Tätigkeitsgruppen und Berufe aus dem Professionsstatus aus. Bereits Hartmann bemerkte 1968, dass es problematisch sei, den Professionsbegriff anhand traditioneller Professionen zu entwickeln und dann als Grundlage für die Beurteilung moderner Professionalisierung zu verwenden (Hartmann 1968). Laut Meyer (2000) ist eine vollständige Autonomie einer Berufsgruppe in der Realität kaum anzutreffen, gleichzeitig impliziere die Einschränkung des Professionsstatus auf rein klientInnenorientierte Tätigkeiten 
eine Nicht-Anwendbarkeit des Professionsbegriffs auf viele moderne Berufsgruppen. Beruf und Profession in ihrer historischen Form seien aufgrund realer Veränderungen der sozialen Wirklichkeit der Organisation von Arbeit ,immer weniger geeignet, diese veränderte soziale Realität zu beschreiben, ohne dass dies jedoch gleichzeitig das Ende von Beruflichkeit oder von Professionalität bedeuten muss“" (Meyer 2000: 88). Meuser (2005b) geht weiter und schlägt vor, die Erforschung professionellen Handelns von der Professionssoziologie abzulösen, da man mithilfe der Professionskriterien nicht fassen könne, was in den unterschiedlichen Handlungsfeldern unter professionellem Handeln verstanden werde. So gebe es ,professionelles Handeln außerhalb von Professionen, allerdings nicht unabhängig von Expertenwissen“ (Meuser 2005b: 261).

Um auch Entwicklungsverläufe derjenigen Berufsgruppen zu erfassen, die einen Professionsstatus nicht erreichen, entwickelten einige AutorInnen dynamische Modelle der Professionalisierung. Hartmann (1968) beschreibt ein Konzept, das eine starre Zuordnung zu den Kategorien Arbeit, Beruf und Profession vermeidet und stattdessen die Prozesse der Verberuflichung und Professionalisierung in den Vordergrund stellt. Während sich Verberuflichung auf den Übergang von Arbeit zum Beruf bezieht, wird der Prozess vom Beruf zur Profession als Professionalisierung verstanden. Dieser erfolgt anhand zweier Dimensionen: Wissen und Sozialorientierung. Innerhalb der Wissensdimension ist der Prozess durch die Herausbildung von kausal differenziertem und ursächlichem Wissen gekennzeichnet, was Hartmann auch als „Verwissenschaftlichung“ (1968: 202) bezeichnet, da dabei die Ausrichtung auf Forschungsergebnisse zunimmt. Die Dimension der sozialen Orientierung bezieht sich auf eine zunehmende Ausrichtung und ein wachsendes Bewusstsein gegenüber der (eigenen) beruflichen Leistung für die Gesellschaft. Ein weiteres prozesshaftes Modell entwickelte Offerhaus (2011) zur Anwendung auf den EU-Journalismus. Laut Offerhaus vollzieht sich der Prozess der Professionalisierung in den beiden Dimensionen Expertisierung und Inszenierung. Unter Expertisierung versteht sie die Herausbildung einer berufsspezifischen Handlungskompetenz. Diese geht ebenfalls mit einer Verwissenschaftlichung einher. Im Zuge der Expertisierung kommt es zu einer Inszenierung, ein Konzept, das auf Pfadenhauer (2005) zurückgeht und besagt, dass ein ExpertInnenstatus nur erlangt werden kann, wenn eine Berufsgruppe als zuständig und kompetent für einen bestimmten Bereich anerkannt wird. Professionalität benötigt hier quasi erst eine soziale Etikettierung. Diese Inszenierung macht es schließlich einer Berufsgruppe möglich, auch unabhängig von Lizenzen eine Autonomie und Anerkennung durch Dritte zu erlangen. Laut diesem Verständnis von Professionalität ist eine legitimierte Definitionsmacht für ein thematisches Feld - anders als im traditionellen Professionsverständnis - auch ohne staatliche Zertifizierung möglich. Ohne das Vorhandensein einer berufsspezifischen Expertise würde eine Inszenierung die Berufsgruppe allerdings langfristig abwerten, da eine Abgrenzung zu LaiInnen nicht mehr gegeben wäre und somit der ExpertInnenstatus auf Dauer nicht glaubwürdig aufrechterhalten werden könnte (Offerhaus 2011). Das ExpertInnenwissen ist sowohl bei den eben vorgestellten dynamischen Modellen als auch bei den modernen professionssoziologischen Ansätzen generell ein zentrales Element der Professionalisierung. Es umfasst berufsspezifisches Wissen (Sach- und Fachwissen) und berufsspezifische Handlungskompetenzen (Erfahrungswissen). Zentral ist dabei die Ausrichtung an aktuellen Forschungsergebnissen (Meuser 2005b; Offerhaus 2011). 


\subsection{Expertisierung der Gleichstellungsarbeit}

Die eben beschriebenen Konzepte ermöglichen es, Professionalisierungsprozesse in der hochschulischen Gleichstellungsarbeit zu untersuchen. Im vorliegenden Beitrag liegt das Augenmerk auf der Expertisierung der Gleichstellungsarbeit, da sie als zentrales Kriterium einer Professionalisierung herausgearbeitet werden konnte. ${ }^{4}$ Nach Hartmann (1968) und Offerhaus (2011) ist eine wachsende Nähe zu Wissenschaft und Forschung ebenso relevant wie eine zunehmend standardisierte Anwendung von praxisbezogenem und problemlösungsorientiertem Wissen. Die Implementierung der Frauen- und Geschlechterforschung schafft eine potenzielle wissenschaftliche Basis für die Gleichstellungsarbeit, die abstraktes und ursächliches Wissen zur Verfügung stellt. Allerdings existiert bis dato keine bewährte Strategie, die Inhalte theoretischen Geschlechterwissens für die Gleichstellungspraxis konkret nutzbar zu machen, um daraus wiederum ein einheitliches Profil von praxisbezogenem Wissen und entsprechenden Handlungskompetenzen zu entwickeln. Laut Wetterer ist dies den qualitativ unterschiedlichen „Spielarten von Geschlechterwissen“ (Wetterer 2009b: 48) geschuldet, die sich in den letzten Jahren aufgrund des Wandels der Gleichstellungspolitik weiter ausdifferenziert haben (Wetterer 2009a, 2009b). Die beiden Wissensarten - das wissenschaftliche Geschlechterwissen feministischer TheoretikerInnen und das GenderexpertInnenwissen von gleichstellungspolitischen AkteurInnen - ergeben sich aus den unterschiedlichen Handlungslogiken und Anforderungen der jeweiligen Bereiche, in denen sie Anwendung finden. ${ }^{5}$ GenderexpertInnen können laut Wetterer (2009b) wissenschaftliches Wissen nicht einfach in der Praxis anwenden, sondern müssen es im Hinblick auf die spezifische Praxis reformulieren und neu konzipieren. Diese Neukonzeption orientiert sich allerdings an den Handlungszielen ihrer Klientel, die wiederum mit den konstruktivistischen Ansätzen der feministischen Theorien meist wenig vertraut sind, sondern sich am Alltagsverständnis der Zweigeschlechtlichkeit orientieren.

In der Diskussion um den Wandel und die Professionalisierung von GenderexpertInnen ist bisher unklar, wie wissenschaftliches Geschlechterwissen in die Praxis der Gleichstellungsarbeit integriert werden kann (Riegraf/Zimmermann 2005; Riegraf 2008, 2009; Wetterer 2007, 2009a, 2009b; Meuser 2009). Riegraf und Vollmer (2014) plädieren dabei für eine engere Kommunikation zwischen Wissenschaft und außerwissenschaftlicher Praxis, die Anerkennung und Wahrung von Distanz zwischen den unterschiedlichen Wissenstypen zu Geschlecht sowie für eine vorherige Ausformulierung von Qualitätskriterien und Standards für Genderkompetenz. Die Entwicklung und Vermittlung solcher Kriterien und Standards könne als Indikator einer Professionalisierung

4 Zieht man die Hartmann'sche Dimension der Sozialorientierung heran, nach der sich eine Professionalisierung durch einen Übergang von der Ausrichtung des beruflichen Handelns auf die eigenen Bedürfnisse bis zur Ausrichtung an einem höheren gesellschaftlichen Wert auszeichnet (Hartmann 1968), so müsste man der Gleichstellungspolitik im Allgemeinen eine fortgeschrittene Professionalisierung zurechnen, da sie dem Leitziel einer Chancengleichheit von Männern und Frauen in der Gesellschaft folgt. Die hochschulische Gleichstellungsarbeit soll zunächst die Chancengleichheit an der Hochschule, sprich auf der Organisationsebene, herstellen und hätte damit laut Hartmann zumindest den Übergang von Arbeit zum Beruf erreicht.

5 Der dritte Wissenstyp nach Wetterer (2009b), das alltagsweltliche Wissen der Gesellschaftsmitglieder, bleibt hier außen vor, da er nicht im Fokus der Betrachtung steht. 
der Gleichstellungsarbeit gesehen werden. Welche Bemühungen im Hinblick auf die Integration von wissenschaftlichem Geschlechterwissen in die Gleichstellungspraxis bereits unternommen werden und welcher Bedarf nach mehr wissenschaftlicher Nähe bei den GleichstellungspraktikerInnen überhaupt besteht, wurde bisher nicht systematisch untersucht. Strebt man eine Verbesserung des Theorie-Praxis-Transfers an, ist die Kenntnis des Status quo allerdings unerlässlich, um Anknüpfungspunkte eines solchen Transfers zu finden. Einen ersten Aufschluss - zumindest in Bezug auf die Gleichstellungsarbeit an deutschen Hochschulen - liefert das nächste Kapitel.

\section{Geschlechterwissen in der hochschulischen Gleichstellung - Ergebnisse zweier Erhebungen}

Die Ergebnisse der im Folgenden vorgestellten Erhebungen sollen die Chancen einer Expertisierung der hochschulischen GleichstellungsakteurInnen aufzeigen. Thematisiert wird dabei die Verbreitung theoretischen Geschlechterwissens, dessen Anwendung in der Praxis sowie die Verknüpfung dieses Wissens mit Gleichstellungsexpertise. Dabei wird verglichen, wie sich die AkteurInnen mit geschlechtertheoretischem Wissen von denjenigen ohne solch einen Hintergrund unterscheiden. Hierfür wird auf Ergebnisse einer quantitativen Online-Befragung und einer qualitativen Interviewstudie mit hochschulischen Amts- und StelleninhaberInnen der Gleichstellungsarbeit Bezug genommen. Die Erhebungen wurden im Rahmen des HGiW-Projektes in den Jahren 2011/2012 durchgeführt. ${ }^{6}$

\subsection{Befragung der Amts- und StelleninhaberInnen}

Die Stichprobe für die Online-Befragung umfasste alle in der hochschulischen Gleichstellungsarbeit tätigen Amts- und StelleninhaberInnen und wurde anhand einer Personenrecherche mithilfe der Websites der Hochschulen in Deutschland zusammengestellt. Aus den Recherchen konnte ein Gesamtsample von 1251 Personen gebildet werden. Dazu zählten neben Gleichstellungsbeauftragten MitarbeiterInnen in Gleichstellungsbüros, in gleichstellungsbezogenen Stabsstellen sowie weiteren organisatorischen Einheiten, die mit Gleichstellung befasst sind. Der finale Datensatz, der für die Auswertung genutzt wurde, beinhaltet 472 Fälle und ergibt damit eine Rücklaufquote von 38 Prozent. Die Stichprobe setzt sich zu etwa gleichen Teilen aus Gleichstellungsbeauftragten (51,1 Prozent) und wissenschaftlichen sowie nicht-wissenschaftlichen MitarbeiterInnen aus dem Bereich der Gleichstellung (48,9 Prozent) zusammen. Die Auswertung erfolgte überwiegend deskriptiv. Bei der Interpretation der Daten muss berücksichtigt werden, dass es für die verschiedenen Akteursgruppen, sprich hauptamtliche und freigestellte Gleichstellungsbeauftragte ${ }^{7}$ sowie GleichstellungsakteurInnen innerhalb und außerhalb

6 Eine ausführliche Darstellung der Ergebnisse ist nachzulesen in LötherNollmer (2014).

7 Das Amt der Gleichstellungsbeauftragten kann je nach Bundesland durch Mitglieder der Hochschule oder auch durch externe Bewerberlnnen besetzt werden. Bei einer Besetzung durch Hoch- 
von Gleichstellungsbüros, unterschiedliche Anforderungen bei Amts- und Stellenbesetzungsverfahren sowie dem Anteil der für Gleichstellungsarbeit verwendeten Arbeitszeit gibt.

Zunächst ist von Interesse, wie verbreitet theoretisches Geschlechterwissen unter den hochschulischen GleichstellungsakteurInnen ist. Insgesamt haben fast die Hälfte der Befragten (46 Prozent) Frauen- und Geschlechterforschung in ihrer Bildungsbiografie behandelt und über ein Drittel (35 Prozent) betreibt weiterhin Geschlechterforschung. Dabei sind teilweise erhebliche Unterschiede je nach Akteursgruppe zu finden. Während 70 Prozent der hauptamtlichen Gleichstellungsbeauftragten einen Bezug zur Frauen- und Geschlechterforschung aufweisen können, sind es bei den Gleichstellungsbeauftragten in Freistellung nur 22 Prozent. Es ist also durchaus theoretisches Geschlechterwissen bei GleichstellungspraktikerInnen vorhanden, das allerdings stark zwischen den Akteursgruppen variiert.

Die Ergebnisse der Online-Erhebung zeigen außerdem diverse Unterschiede zwischen den Befragten mit und ohne geschlechtertheoretischen Hintergrund. Von Interesse war, welche Bedeutung die Befragten dem theoretischen Wissen für die Praxis geben und wie sie die eigene Expertise wahrnehmen. Außerdem sollte ermittelt werden, inwiefern die Gleichstellungsarbeit für die Befragten den Charakter eines Berufs hat. Um zu erfahren, welche Bedeutung dem theoretischen Wissen für die Praxis zugesprochen wird, wurden die Befragten darum gebeten, einzuschätzen, welche Rolle Frauen- und Geschlechterforschung für ihre Tätigkeit in der Gleichstellung spielt. Die Einschätzung variiert hier je nach eigenem geschlechtertheoretischen Hintergrund. Von den Befragten mit Bezug zur Frauen- und Geschlechterforschung geben fast 70 Prozent an, dass die geschlechtertheoretischen Kenntnisse hilfreich oder sehr hilfreich für ihre Arbeit in der Gleichstellung sind. Dagegen geben nur 26 Prozent der Befragten ohne solch einen theoretischen Bezug an, dass diese Art von Wissen für ihre Arbeit hilfreich gewesen wäre. GleichstellungspraktikerInnen, die Frauen- und Geschlechterforschung behandelt haben, schätzen diese als bedeutender für die Gleichstellungspraxis ein als diejenigen, die sich kein theoretisch-wissenschaftliches Geschlechterwissen während ihrer Bildungsbiografie angeeignet haben. Je nach eigenem theoretischen Bezug schätzen die Befragten die eigene Expertise unterschiedlich ein. Sie wurden darum gebeten, eine Einschätzung ihrer Selbstwahrnehmung als GleichstellungsexpertIn mithilfe einer Fünfstufenskala ${ }^{8}$ vorzunehmen. Insgesamt sehen sich die meisten Befragten nur bedingt als ExpertInnen in Sachen Gleichstellung. Diejenigen Befragten, die Inhalte der Frauenund Geschlechterforschung im Studium hatten, stufen sich höher ein als die Befragten ohne entsprechenden Hintergrund. Unterschiede gab es auch bei der Wahrnehmung beruflicher Perspektiven und der wöchentlichen Arbeitszeit. So geben AkteurInnen mit einem geschlechtertheoretischen Hintergrund deutlich häufiger an, sich bewusst für eine Tätigkeit in der Gleichstellung entschieden zu haben, sie schätzen berufliche Perspek-

schulmitglieder kann die Gleichstellungsbeauftragte bis zu einem gewissen Grad von ihren dienstlichen Pflichten freigestellt werden. Bei einer Besetzung durch hochschulexterne Personen handelt es sich um ein hauptamtliches bzw. hauptberufliches Amt.

8 Die Befragten wurden gebeten, zu den folgenden beiden Aussagen den Grad ihrer Zustimmung auf einer Skala von 1 (stimme überhaupt nicht zu) bis 5 (stimme voll und ganz zu) anzugeben: „Ich bin eine Expertin/ein Experte in Sachen Gleichstellung"; "Meine Expertise zum Thema Gleichstellung wird häufig nachgefragt". 
tiven in diesem Arbeitsbereich optimistischer ein und arbeiten mehr Wochenstunden in der Gleichstellungsarbeit als Befragte ohne einen solchen Hintergrund. Es zeigt sich also ein positiver Zusammenhang zwischen wissenschaftlichem Wissen und Professionalisierungskriterien. In welchem Umfang das wissenschaftliche Geschlechterwissen dabei behandelt und studiert wurde, ist aus diesen Daten nicht erkennbar, insofern sind die Unterschiede zwischen denjenigen mit und ohne solch einen theoretischen Hintergrund stets mit Vorsicht zu interpretieren.

Die wöchentliche Arbeitszeit ist eine entscheidende Variable ${ }^{9}$ bei der Betrachtung von Professionalisierungskriterien. So gibt es einen positiven Zusammenhang zwischen Arbeitszeit und Bezug zur Frauen- und Geschlechterforschung einerseits und zwischen Arbeitszeit und Selbsteinschätzung als ExpertIn andererseits. Hier muss allerdings das Amtsmodell der Gleichstellungsbeauftragten als erklärende Variable berücksichtigt werden, da Gleichstellungsbeauftragten im Freistellungsmodell durchschnittlich deutlich weniger wöchentliche Arbeitszeit zur Verfügung steht als ihren hauptamtlichen KollegInnen. ${ }^{10}$ Gleichzeitig geben die freigestellten Gleichstellungsbeauftragten im Vergleich zu allen anderen befragten AkteurInnen deutlich seltener an, Inhalte der Frauen- und Geschlechterforschung als Teil des Studiums behandelt zu haben.

Weil es für Gleichstellungsarbeit keine allgemeingültigen formalen Ausbildungsanforderungen gibt, sind für den Aufbau einer gemeinsamen Wissensbasis und Expertise von GleichstellungspraktikerInnen „Learning on the Job“ bzw. die Strategien der Informationsbeschaffung von großer Bedeutung. Die AkteurInnen wurden zu diesem Zweck danach gefragt, auf welche Weise sie sich über neue Entwicklungen, Best Practices etc. im eigenen gleichstellungsbezogenen Arbeitsbereich informieren. Zur Auswahl standen diverse Strategien der Informationsbeschaffung, die in ihrem Grad der Formalität variierten. Die Befragten verwenden vor allem informelle Strategien wie den persönlichen Austausch mit GleichstellungsakteurInnen der eigenen oder anderer Hochschulen. Formellere und institutionalisierte Strategien wie die Teilnahme an Weiterbildungsangeboten oder Coachings werden eher selten in Anspruch genommen. Gleichstellungsbeauftragte nutzen dabei die Vernetzung mit anderen Hochschulen, auch durch die Bundeskonferenz und Landeskonferenzen der Frauen- und Gleichstellungsbeauftragten (BuKoF, LaKof), stärker im Vergleich zu anderen AkteurInnen in der Gleichstellungsarbeit, die keinen Zugang zur formalen Mitgliedschaft in diesen Vereinigungen haben. Derzeit gibt es keine Netzwerke, die allen hochschulischen GleichstellungsakteurInnen eine formale Mitgliedschaft und Vertretung nach außen ermöglichen.

Die Ergebnisse der Online-Befragung zeigen, dass bei einer großen Gruppe der befragten GleichstellungsakteurInnen ein Bezug zu theoretisch-wissenschaftlichem Geschlechterwissen vorhanden ist und dass die AkteurInnen diese Kenntnisse für wichtig erachten und bemüht sind, sie weiter auszubauen. Auch sehen sie sich eher als GleichstellungsexpertInnen und schätzen ihre berufliche Perspektive in der Gleichstellungsarbeit im Vergleich zur Untersuchungsgruppe ohne fachlichen Bezug zur Frauen- und Geschlechterforschung optimistischer ein. Die genannten Variablen - der theoretische

9 Gefragt wurde an dieser Stelle nach der vertraglich festgelegten Arbeitszeit.

10 Hauptamtliche Gleichstellungsbeauftragte verwenden vertraglich festgelegt im Durchschnitt 30 Wochenstunden für die Gleichstellungsarbeit an ihrer Hochschule, während es bei den Gleichstellungsbeauftragten im Freistellungsmodell durchschnittlich nur 11 Wochenstunden sind. 
Bezug, die Selbstbeurteilung der Gleichstellungsexpertise und der Grad der Orientierung an den Ergebnissen der Geschlechterforschung - korrelieren positiv mit einer höheren wöchentlichen Arbeitszeit in der Gleichstellungspraxis. Um sich Informationen anzueignen, die für den eigenen Arbeitsbereich in der Gleichstellung relevant sind, greifen die AkteurInnen vor allem auf informelle Strategien zurück, wobei Gleichstellungsbeauftragte hochschulübergreifend besser vernetzt sind als MitarbeiterInnen. Berücksichtigt werden müssen stets die unterschiedlichen Rahmenbedingungen von Gleichstellungsbeauftragten und MitarbeiterInnen auf der einen sowie hauptamtlichen und freigestellten Gleichstellungsbeauftragten auf der anderen Seite. Vor allem Gleichstellungsbeauftragte im Freistellungsmodell weisen hier im Vergleich zu den anderen Akteursgruppen eine geringere Expertisierung auf.

Aus den Daten der quantitativen Erhebung wird nicht deutlich, welcher konkrete Nutzen dem theoretischen Wissen für die Praxis durch die Befragten zugesprochen wird und wie das theoretische Wissen in der Praxis bisher zur Anwendung kommt. Hier geben die Ergebnisse der qualitativen Interviews mehr Aufschluss anhand exemplarischer Fälle.

\subsection{Qualitative Fallstudien}

Für die qualitative Erhebung wurden anhand einer zuvor durchgeführten Bestandserhebung der Ämter und Stellen in der hochschulischen Gleichstellungsarbeit fünf Fallhochschulen ausgewählt. Dabei handelte es sich um vier Universitäten und eine Hochschule für angewandte Wissenschaften. Auswahlkriterien waren neben der regionalen Verteilung das Vorhandensein eines Gleichstellungsbüros und gleichstellungsbezogene Stellen außerhalb des Gleichstellungsbüros. An den Fallhochschulen wurden jeweils leitfadengestützte telefonische Interviews mit der zentralen Gleichstellungsbeauftragten, einer Mitarbeiterin des Gleichstellungsbüros und einer Gleichstellungspraktikerin außerhalb des Gleichstellungsbüros durchgeführt. Die Stichprobe der vorliegenden Auswertung umfasst 16 Personen. ${ }^{11}$ Die Interviews thematisierten unter anderem den Begriff Genderexpertise und die Bedeutung wissenschaftlichen Geschlechterwissens für die Gleichstellungspraxis. Die Auswertung der transkribierten Interviews erfolgte nach der Methode der qualitativen Inhaltsanalyse nach Mayring (2002).

Die Befragten wurden zum einen darum gebeten, ihre persönliche Auffassung des Begriffs Genderexpertise zu geben und zum anderen die Bedeutung von Frauen- und Geschlechterforschung für die Gleichstellungspraxis, insbesondere ihren eigenen Arbeitsbereich, einzuschätzen. Auffallend ist dabei, dass diejenigen Interviewpartnerinnen mit eigenem Bezug zu theoretischem Geschlechterwissen diesem auch eine höhere Bedeutung für Expertise und Praxis beimessen und umgekehrt. Die Akteurinnen orientierten sich bei den Einschätzungen häufig an ihren eigenen Kompetenzen.

Der Begriff Genderexpertise wird von den meisten Interviewpartnerinnen mit einem theoretischen Hintergrundwissen der Frauen- und Geschlechterforschung auf der einen und mit langjähriger Erfahrung in der Gleichstellungsarbeit auf der anderen Seite

11 Zusätzlich wurden Interviews mit einem Mitglied des Rektorats bzw. Präsidiums der jeweiligen Fallhochschule durchgeführt. Diese sind für die Fragestellung des vorliegenden Beitrags jedoch nicht relevant und werden daher bei der Darstellung der Auswertung nicht berücksichtigt. 
assoziiert. Die Mitarbeiterin eines Gleichstellungsbüros äußert sich wie folgt zum Begriff (Interview Nr. 12):

„Also, für mich [...] heißt es tatsächlich, [...] Expertise ist dann da, wenn man sich [...] wirklich schon sehr lange mit einem Thema beschäftigt, in dem Fall eben Gleichstellungsarbeit. [...] Erst dann kann man von Expertise reden. Nichtsdestotrotz glaube ich, dass auch das Training on the Job (lachend), wie es so schön heißt, [...], ja, doch irgendwie zu einer gewissen Professionalisierung führt. Aber das Expertinnen- und Experten-Dasein, das würde ich jetzt denjenigen zusprechen, die sich wirklich schon lange damit beschäftigen. Das heißt also, die, ja, im Gender-Studies-Bereich [...], ja, sich schon lange bewegen. [...] Ja".

Ob der theoretische Hintergrund oder die langjährige praktische Erfahrung im Vordergrund steht, hängt bei den Befragten häufig eng mit dem eigenen Erfahrungshintergrund zusammen. Die genannten Kriterien für Genderexpertise orientieren sich dabei häufig an den Anforderungen der jeweils eigenen Stelle bzw. des Amtes. So betonen diejenigen Befragten, die sich bereits seit ihrem Studium mit Frauen- und Geschlechterforschung beschäftigen und heute als MitarbeiterInnen im Gleichstellungsbüro oder einer Stabsstelle mit Genderthematik tätig sind, den theoretischen Aspekt etwas stärker, während Gleichstellungsbeauftragte ohne geschlechtstheoretischen Hintergrund oder langjährige Tätigkeit in der Gleichstellungsarbeit eher den praktischen Aspekt der Genderexpertise in den Vordergrund stellen.

Die Relevanz von Frauen- und Geschlechterforschung für Genderexpertise wird von der Mehrheit der Befragten als hoch eingeschätzt, wobei dafür unterschiedliche Gründe angeführt werden und auch die Verwertbarkeit für die tägliche Arbeit unterschiedlich beurteilt wird. Kenntnisse der Frauen- und Geschlechterforschung dienen zum einen als Argumentationsgrundlage in der Kommunikation mit HochschulakteurInnen, insbesondere bei Verhandlungen mit der Hochschulleitung. Bezüglich der Bedeutung dieses Wissens für die Praxis werden vor allem zwei verschiedene Meinungen vertreten. Ein Teil der Befragten betont, dass Frauen- und Geschlechterforschung die theoretische Grundlage oder Grundlagenforschung für die praktische Gleichstellungsarbeit darstellt, anhand derer strategische Konzepte erarbeitet werden können, wie es beispielsweise die Leiterin einer Stabsstelle für Gleichstellung und Diversity im Interview ausführt (Interview Nr. 14):

"[...] weil ich der Meinung bin, dass das wirklich WICHTIG ist als Grundlage und man eben NICHT einfach davon ausgehen kann, dass JEDER Gleichstellung kann. [...] Mittlerweile ist das Feld [...] sehr gewachsen, sehr verästelt, also, wirklich, hat sich echt weiterentwickelt. Viele Expertisen, die da sind, und wenn sie das nicht wissen und KENNEN, dann agieren sie immer aus dem Bauch raus. [...] Die sind dann irgendwie so ein bisschen wie freischwebende Satelliten unterwegs [... $]^{\prime \prime}$.

Hier wird eine theoretisch-wissenschaftliche Fundierung von GleichstellungsakteurInnen als Indikator genommen, um GleichstellungsexpertInnen von -laiInnen zu unterscheiden. Gerade angesichts der thematischen und strukturellen Ausdifferenzierung der Gleichstellungsarbeit sieht die Befragte im frauen- und geschlechterwissenschaftlichen Hintergrund der AkteurInnen das Potenzial, als eine Art Gütekriterium für wissenschaftlich fundierte und nicht intuitiv ,,aus dem Bauch raus“ praktizierte Gleichstellungsarbeit zu fungieren. 
Die andere, in den Interviews weniger oft vertretene Meinung, ist das Befürworten der Abgrenzung von wissenschaftlich-theoretischem Geschlechterwissen bzw. feministischer Rhetorik und Gleichstellungspraxis. Hierzu beispielhaft folgender Interviewauszug mit einer weiteren Leiterin einer Stabsstelle für Gleichstellung und Diversity (Interview Nr. 3):

\footnotetext{
"Aber gleichzeitig [...] darf man es trotzdem nicht zu sehr propagieren, weil das genau [...], ja es gibt ja so ein paar No-Gos in der Arbeit, in der Gleichstellungsarbeit. Und wenn man zu sehr mit Gender, Genderthemen kommt, Genderdaten, Fakten, ich weiß nicht was. Dann ist man ja da direkt gleich in Verruf, beziehungsweise, ja, das ist [...], da wenden sich die Leute ab".
}

Bei einigen Befragten kam die Sorge zum Vorschein, Mitglieder der Hochschulleitung durch allzu „hartes“ feministisches oder in der Frauen- und Geschlechterforschung gängiges Vokabular zu verschrecken. Das feministisch-theoretische Wissen wurde in diesen Fällen als untauglich eingestuft. Es sei nicht anwendbar auf die alltägliche Arbeit der jeweiligen Befragten und nicht vereinbar mit dem „,modernen“ Gleichstellungsgedanken, der auch Männern eine Teilhabe an der Gleichstellungspolitik ermöglichen soll.

Die Befragten der Interviewstudie haben unterschiedliche Auffassungen über die Bedeutung des Begriffs Genderexpertise und messen dem geschlechtertheoretischen Wissen einen unterschiedlichen, wenn auch insgesamt hohen Stellenwert zu. Meist spielen für die Einschätzungen die Anforderungen und Erfahrungen des jeweils eigenen Arbeitsbereichs sowie der eigene fachliche Hintergrund eine entscheidende Rolle. Die Aussagen der Interviewten sind dabei auch innerhalb einer Fallhochschule heterogen. Die Bedeutung der Frauen- und Geschlechterforschung für die Praxis der Gleichstellungsarbeit wird überwiegend als groß eingestuft. Die meisten AkteurInnen versuchen, sich wissenschaftliches Geschlechterwissen für ihre Arbeit zunutze zu machen. Dabei gehen sie nach eigenem Ermessen und nach eigenen inhaltlichen Schwerpunktsetzungen vor, da es an keiner der Fallhochschulen eine institutionalisierte Form gibt, theoretisch-wissenschaftliches Wissen für die Praxis zu nutzen. Deutlich wird auch, dass sich manche Befragte einem Drahtseilakt gegenüber sehen: Einerseits soll wissenschaftliches Wissen als Grundlagenwissen zur Untermauerung der Notwendigkeit von gleichstellungspolitischen Maßnahmen vor allem im Rahmen von Aushandlungsprozessen mit der Hochschulleitung bereitstehen. Andererseits sollen diejenigen AkteurInnen, die es noch von der Thematik zu überzeugen gilt, nicht mit theoretisch-feministischen Begrifflichkeiten überfordert werden.

Sowohl in der quantitativen als auch in der qualitativen Untersuchung schätzen diejenigen AkteurInnen mit einem geschlechterwissenschaftlichen Hintergrund die Bedeutung der Theorie für die Praxis im Vergleich zu ihren KollegInnen ohne diesen Hintergrund höher ein. Zwar wird in der Frauen- und Geschlechterforschung ein wichtiges Potenzial für die Gleichstellungspraxis gesehen, ob und wie deren Inhalte für den eigenen Arbeitsalltag genutzt werden, bleibt den AkteurInnen allerdings selbst überlassen. Auch weist die Heterogenität der Aussagen der Befragten innerhalb der Fallhochschulen auf die Abwesenheit von Standards oder Leitlinien zur Nutzbarmachung von wissenschaftlichem Wissen für die Gleichstellungsarbeit hin. 


\section{$4 \quad$ Fazit}

Gleichstellungspolitische AkteurInnen haben heute die Möglichkeit - dank der Verbreitung der Frauen- und Geschlechterforschung - auf wissenschaftliches Geschlechterwissen zurückzugreifen. Die Ergebnisse der beiden Erhebungen weisen einerseits auf existierende geschlechtertheoretische Untermauerung und Nutzung von theoretischem Geschlechterwissen hin, andererseits werden aber auch die Willkür der Nutzung und des Ausbaus dieses Wissens sowie die Ambivalenz gegenüber der Relevanz für die Praxis deutlich. Nach über 30 Jahren Gleichstellungsarbeit an deutschen Hochschulen dürfte es außerdem einen beachtlichen Pool an praxisbezogenem Erfahrungswissen geben. Jedoch existiert weder für das Erschließen und Nutzen dieses Wissens noch des wissenschaftlichen Geschlechterwissens ein bewährtes System. Anhand der Ergebnisse der hier genannten Erhebungen wird deutlich, dass GleichstellungspraktikerInnen vor allem informelle Informations- und Austauschstrategien verwenden und sich ihre für die Gleichstellungspraxis benötigten Kompetenzen nach eigenem Ermessen aneignen. Dementsprechend sind die Unterschiede zwischen den AkteurInnen in der Gleichstellungsarbeit hinsichtlich erfahrungs- und praxisbezogenem Wissen, feministisch-theoretischem bzw. wissenschaftlichem Geschlechterwissen sowie Problembewusstsein und Auffassungen gegenüber gleichstellungspolitischen Themen sehr groß. Für die weitere Professionalisierung der hochschulischen Gleichstellungsarbeit ist die Entwicklung in Richtung einer gemeinsamen Expertise und Wissensbasis eine wichtige Voraussetzung. Chancen sind hier im Ausbau von formellen und institutionalisierten Weiterbildungsmöglichkeiten und in einer übergreifenden Anbindung der GleichstellungsakteurInnen in institutionalisierten Netzwerken zu sehen.

Inwiefern allein die Frauen- und Geschlechterforschung als theoretisch-wissenschaftliches Wissen für die Gleichstellungsarbeit tauglich ist, sei an dieser Stelle offengelassen, denn auch Disziplinen und Fachrichtungen wie die Rechtswissenschaften, Organisationssoziologie oder - angesichts der Implementierung des New Public Managements - eventuell auch Wirtschaftswissenschaften könnten nutzbare Inhalte für die Arbeit von GleichstellungspraktikerInnen bereithalten. Die Strategien zur Verwertung dieses Wissens müssen unter Berücksichtigung des Arbeitsalltags und der heterogenen Arbeitsinhalte einerseits sowie der Erkenntnisse der herangezogenen wissenschaftlichen Disziplinen andererseits erarbeitet werden.

Wie bereits anfangs erwähnt, vertritt dieser Beitrag eine analytische Perspektive, indem sich die Autorinnen am deskriptiv-analytischen Verständnis des Professionalisierungsbegriffs nach Kleimann (2011) orientieren. Diese Perspektive ist für eine professionssoziologische Betrachtung der aktuellen Entwicklungen in der Gleichstellungspolitik insofern sinnvoll, als sie die Abgrenzung von normativen Aussagen im Sinne ,professioneller' und ,unprofessioneller' Gleichstellungsarbeit ermöglicht. Dem Streben der Gleichstellungsarbeit nach Aufwertung kann diese Betrachtung allerdings auch Chancen aufzeigen, da so z. B. Vergleiche mit Entwicklungsprozessen anderer Tätigkeits- bzw. Berufsgruppen möglich sind. 


\section{Literaturverzeichnis}

Blome, Eva; Erfmeier, Alexandra; Gülcher, Nina \& Smykalla, Sandra. (2013). Handbuch zur universitären Gleichstellungspolitik. Von der Frauenförderung zum Diversity Management? (2. Aufl.). Wiesbaden: VS Verlag.

Evetts, Julia. (2003). The Sociological Analysis of Professionalism. Occupational Change in the Modern World. International Sociology, 18(2), 395-415.

Hartmann, Heinz. (1968). Arbeit, Beruf, Profession. Soziale Welt, 19(19), 193-216.

Kleimann, Bernd. (2011). Professionalisierung der Hochschulleitung? In Andreas Langer \& Andreas Schröer (Hrsg.), Professionalisierung im Nonprofit Management (S. 201-226). Wiesbaden: VS Verlag.

Krücken, Georg; Blümel, Albrecht \& Kloke, Katharina. (2010). Hochschulmanagement - auf dem Weg zu einer neuen Profession? WSI Mitteilungen, (5), 234-241. Zugriff am 12. Dezember 2012 unter www.boeckler.de/wsimit_2010_05_kruecken.pdf.

Löther, Andrea \& Vollmer, Lina. (Hrsg.). (2014). Gleichstellungsarbeit an Hochschulen. Neue Strukturen - neue Kompetenzen. Leverkusen: Barbara Budrich.

Macha, Hildegard; Gruber, Susanne \& Struthmann, Sandra. (2011). Die Hochschule strukturell verändern. Gleichstellung als Organisationsentwicklung an Hochschulen. Opladen: Barbara Budrich.

Mayring, Philipp. (2002): Einführung in die qualitative Sozialforschung (5. Aufl.). Weinheim, Basel: Beltz.

Meuser, Michael. (2005a). Gender-Management. Zur Professionalisierung von Geschlechterpolitik. Supervision: Mensch, Arbeit, Organisation, (2), 14-18.

Meuser, Michael. (2005b). Professionelles Handeln ohne Profession? Eine Begriffskonstruktion. In Michaela Pfadenhauer (Hrsg.), Professionelles Handeln (S. 253-346). Wiesbaden: VS Verlag.

Meuser, Michael. (2009). Humankapital Gender. Geschlechterpolitik zwischen Ungleichheitssemantik und ökonomischer Logik. In Sünne Andresen, Mechthild Koreuber \& Dorothea Lüdke (Hrsg.), Gender und Diversity: Albtraum oder Traumpaar? Interdisziplinärer Dialog zur ,Modernisierung “ von Geschlechter- und Gleichstellungspolitik (S. 95-109). Wiesbaden: VS Verlag.

Meyer, Rita. (2000). Qualifizierung für moderne Beruflichkeit: soziale Organisation der Arbeit von Facharbeiterberufen bis zu Managertätigkeiten. Münster: Waxmann.

Mieg, Harald A. (2003). Problematik und Probleme der Professionssoziologie. In Harald A. Mieg \& Michaela Pfadenhauer (Hrsg.), Professionelle Leistung - Professional Performance. Positionen der Professionssoziologie (S. 11-46). Konstanz: UVK.

Mieg, Harald A. (2006). Professionalisierung. In Felix Rauner (Hrsg.), Handbuch Berufsbildungsforschung (2. aktualisierte Aufl.) (S. 343-350). Bielefeld: Bertelsmann.

Nigges-Gellrich, Anna. (2008). Neue Wege statt alter Pfade. Frauen- und Gleichstellungsarbeit auf dem Weg zur Profession. Hagen, Fernuniversität. (IfBM. Impuls Schriftenreihe des Instituts für Bildungswissenschaft und Medienforschung). Zugriff am 13. November 2009 unter http:// ifbmimpuls.fernuni-hagen.de/2008-2-Frauen-Gleichstellungsarbeit-Profession.pdf.

Offerhaus, Anke. (2011). Die Professionalisierung des deutschen EU-Journalismus. Expertisierung, Inszenierung und Institutionalisierung der europäischen Dimension im deutschen Journalismus. Wiesbaden: VS Verlag. 
Pfadenhauer, Michaela. (2005). Die Definition des Problems aus der Verwaltung der Lösung. Professionelles Handeln revisited. In Michaela Pfadenhauer (Hrsg.), Professionelles Handeln (S. 9-22). Wiesbaden: VS Verlag.

Riegraf, Birgit. (2008). Anwendungsorientierte Forschung und der Wandel der Wissensordnung zu Geschlecht. Konzeptionelle Annäherungen. Österreichische Zeitschrift für Soziologie, (4), $62-78$.

Riegraf, Birgit. (2009). Die Organisation vom Wandel. Gender-Wissen und Gender-Kompetenzen in Wissenschaft und Politik. In Birgit Riegraf \& Lydia Plöger (Hrsg.), Gefühlte Nähe, faktische Distanz. Geschlecht zwischen Wissenschaft und Politik: Perspektiven der Frauenund Geschlechterforschung auf die „Wissensgesellschaft“ (S. 67-80). Opladen: Barbara Budrich.

Riegraf, Birgit \& Vollmer, Lina. (2014). Professionalisierungsprozesse und Geschlechter-Wissen. In Cornelia Behnke, Diana Lengersdorf \& Sylka Scholz (Hrsg.), Wissen - Methode - Geschlecht: Erfassung des fraglos Gegebenen (S. 33-48). Wiesbaden: VS Verlag.

Riegraf, Birgit \& Zimmermann, Karin. (2005). Der Wandel der Wissensordnung in der Wissensgesellschaft und die Kategorie Geschlecht. Annäherungen an ein Forschungsdesiderat. In Maria Funder, Steffen Dörhöfer \& Christian Rauch (Hrsg.), Jenseits der Geschlechterdifferenz? Geschlechterverhältnisse in der Informations- und Wissensgesellschaft (Bd. 5) (S. 21-37). München: Mering.

Schneijderberg, Christian \& Merkator, Nadine. (2011). Hochschulprofessionen und Professionalisierung im Bereich der Qualitätsentwicklung. Qualität in der Wissenschaft (QiW). Zeitschrift für Qualitätsentwicklung in Forschung, Studium und Administration, (1), 15-20.

Schütze, Fritz. (1992). Sozialarbeit als bescheidene Profession. In Bernd Dewe, Wilfried Ferchhoff \& Frank Olaf Radtke (Hrsg.), Erziehen als Profession (S. 132-170). Opladen: Leske + Budrich.

Wetterer, Angelika. (2007). Erosion oder Reproduktion geschlechtlicher Differenzierungen? Zentrale Ergebnisse des Forschungsschwerpunkts ,Professionalisierung, Organisation, Geschlecht' im Überblick. In Regine Gildemeister \& Angelika Wetterer (Hrsg.), Erosion oder Reproduktion geschlechtlicher Differenzierungen? Widersprüchliche Entwicklungen in professionalisierten Berufsfeldern und Organisationen (S. 189-214). Münster: Westfälisches Dampfboot.

Wetterer, Angelika. (2009a). Gender-Expertise, feministische Theorie und Alltagswissen. Grundzüge einer Typologie des Geschlechterwissens. In Birgit Riegraf \& Lydia Plöger (Hrsg.), Gefühlte Nähe, faktische Distanz. Geschlecht zwischen Wissenschaft und Politik: Perspektiven der Frauen- und Geschlechterforschung auf die „Wissensgesellschaft “ (S. 81-99). Opladen: Barbara Budrich.

Wetterer, Angelika. (2009b). Gleichstellungspolitik im Spannungsfeld unterschiedlicher Spielarten von Geschlechterwissen. Eine wissenssoziologische Rekonstruktion. GENDER, 1(2), $45-60$.

Zimmermann, Karin. (2003). Praxis der Gleichstellung - widersprüchliche Modernisierung. Das Beispiel der Hochschulen. Journal Netzwerk Frauenforschung NRW, (15), 55-64. 


\section{Zu den Personen}

Lina Vollmer, Dipl.-Sozialwissenschaftlerin. Arbeitsschwerpunkte: Gleichstellung an Hochschulen, Hochschulmanagement, Governance, Professionssoziologie.

Kontakt: GESIS - Leibniz-Institut für Sozialwissenschaften, Kompetenzzentrum Frauen in Wissenschaft und Forschung CEWS, Unter Sachsenhausen 6-8, 50667 Köln

E-Mail: lina.vollmer@gesis.org

Juliane Mosel, Dipl.-Soziologin. Arbeitsschwerpunkte: Gleichstellung an Hochschulen, Hochschulforschung, AbsolventInnenstudien, qualitative Methoden.

Kontakt: Technische Universität Darmstadt, Hochschuldidaktische Arbeitsstelle, HochschulstraBe 1, 64289 Darmstadt

E-Mail: ju.mosel@gmail.com 